\title{
Fuzzy space-time topology and geometric quantization
}

\author{
S.N. Mayburov ${ }^{1, a}$ \\ ${ }^{1}$ Lebedev Institute of Physics, Moscow, Leninski pr. 53
}

\begin{abstract}
Dodson-Zeeman fuzzy topology considered as the possible mathematical framework of geometric quantum formalism. In such approach the states of massive particle $m$ correspond to elements of fuzzy manifold called fuzzy points. Due to their weak (partial) ordering, $m$ space coordinate $x$ acquires principal uncertainty $\sigma_{x}$. It's shown that $m$ evolution on such manifold is described by Schroedinger or Dirac formalisms in norelativistic and relativistic cases correspondingly.
\end{abstract}

\section{Introduction}

Importance of geometric methods in Quantum Physics is duly acknowledged now, however the reformulation of quantum mechanics (QM) in geometric terms is still an open problem ([1] and refs. therein). Such formalism can be applied primarily for quantum gravity problems, but also for gauge field theory; its implications can be important for the analysis of QM foundations. Currently, the main impact of these studies is done on the exploit of Hilbert manifolds [1], alternatively in the approach considered here the basic structure is the real manifold equipped with fuzzy topology (FT) [2-4]. In connection with such approach it's worth to mention the noncommutative fuzzy spaces with finite (sphere, tori) and infinite discrete structure [5]. The general feature of such theories is that the space coordinates turn out to be principally fuzzy, the reason of that is the noncommutativity of coordinate observables $x_{1,2,3}$. Meanwhile, the similar fuzzy properties can be obtained for the spaces equipped with dedicated FT $[2,3]$. In addition, it was shown that in its framework the quantization procedure by itself can be defined as the transition from the classical phase space to fuzzy one $[6,7]$. Therefore, the quantum properties of particles and fields can be deduced directly from the geometry of phase space induced by underlying FT and don't need to be postulated separately of it. It was shown also that FT equipped geometry induces the geometrodynamics which is equivalent to quantum dynamics $[6,7]$. As the example, the dynamics of massive particles will be considered here. Earlier some phenomenological assumptions were used by in its formulation, here the new formalism which permits to avoid them is exploited [8].

To review FT formalism and its application to QM let's describe it first for the discrete structure and consider some set $A$ of $n$ elements $\left\{a_{i}\right\}$. If it's the ordered set, then the ordering relation between all element pairs $a_{k} \leq a_{l}$ (or vice versa) is fulfilled. But if $A$ is the partial-ordered set (Poset), then some its element pairs can enjoy the incomparability (equivalence) relations (IR) between them: $a_{j} \sim a_{k}$ [9]. If this is the case, then both $a_{j} \leq a_{k}$ and $a_{k} \leq a_{j}$ propositions are false. To illustrate IR meaning, let's choose $A$ such that for it $\forall i, a_{i} \leq a_{i+1}$, except for the single element $a_{0}$ which can be incomparable to

\footnotetext{
ae-mail: mayburov@sci.lebedev.ru
} 
some $a_{j}$. For example, consider some $A$ subset (interval) $\left\{a_{i}, a_{l}\right\}$ and suppose that $a_{0}$ is confined in it, i.e. $a_{i-1} \leq a_{0} \leq a_{l+1}$, yet $a_{0} \sim a_{j}$ for all 'internal' $j$ such that: $i \leq j \leq l$. In this case $a_{0}$ is 'smeared' over $\left\{a_{i}, a_{l}\right\}$ interval, which is the rough analogue of $a_{0}$ coordinate uncertainty if to regard the ordered sequence of $A$ elements as 'coordinate axe' in $A$.

It's possible to detalize such smearing introducing the fuzzy relations, for that purpose one can put in correspondence to each $a_{0}, a_{j}$ pair the weight $w_{j} \geq 0$ with the norm $\sum_{j} w_{j}=1$. In this case $A$ is fuzzy ordered set (Foset), $a_{0}$ called the fuzzy point (FP) [3, 10]. The continuous 1-dimensional Foset $C^{F}$ is defined analogously: $C^{F}=A^{F} \cup X$, where $A^{F}$ is discrete FP subset $\left\{a_{i}\right\}, X$ is continuous ordered subset, which is equivalent to $R^{1}$ axe of real numbers. Correspondingly, fuzzy relations between elements $a_{j}, x$ are described by real function $w^{j}(x) \geq 0$ with the norm $\int w^{j} d x=1$. In 1 dimensional Euclidean geometry, the elements of its manifold $X$ are the points $x_{a}$ which constitute the ordered continuum set. Yet in the geometry with fundamental set $C^{F}$ the position of fuzzy point $a_{j}$ described by the positive normalized function $w^{j}(x)$ on $X$, which called also the fuzzy value $\tilde{x}_{j} ; w^{j}$ dispersion $\sigma_{x}$ characterizes $a_{j}$ coordinate uncertainty on $X$. Note that in such geometry $w^{j}(x)$ doesn't have any probabilistic meaning but only the algebraic one. To describe the distinction between the fuzzy structure and probabilistic one, the weight correlation $K_{0}^{j}\left(x, x^{\prime}\right)$ defined over $w^{j}$ support can be introduced; thus if $w^{j}\left(x_{1,2}\right) \neq 0$, then $\forall x_{1}, x_{2} ; K_{0}^{j}\left(x_{1}, x_{2}\right)=1$ for FP $a_{j}$ and $K_{0}^{j}\left(x_{1}, x_{2}\right)=0$ for probabilistic $a_{j}$ distribution. Thus $a_{j}$ state $g$ on $X$ is described by two functions $g=\left\{w^{j}(x), K_{0}^{j}\left(x, x^{\prime}\right)\right\}$.

\section{Linear Model of Fuzzy Dynamics}

In the described terms the massive particle of 1-dimensional classical mechanics corresponds to the ordered point $x_{a}(t) \in X$. By the analogy, we suppose that in 1-dimensional fuzzy mechanics (FM) constructed here the particle $m$ corresponds to fuzzy point $a(t)$ in $C^{F}$ characterized by normalized positive density $w(x, t)$. Beside $w(x), m$ fuzzy state $\mid g\}$ can also depend on other $m$ degrees of freedom (DFs) characterizing its evolution. The temptative candidate for such DF is $m$ average velocity:

$$
\bar{v}(t)=\frac{\partial}{\partial t} \int_{-\infty}^{\infty} x w(x) d x=\int_{-\infty}^{\infty} x \frac{\partial w}{\partial t}(x, t) d x
$$

It's sensible to suppose that $\bar{v}(t)$ is independent of $w(x, t)$, below we shall also look for such DFs in form of real functions $q_{1, \ldots, n}(x, t)$. Let's suppose that in FM $m$ evolution is local, i.e.:

$$
\frac{\partial w}{\partial t}(x, t)=-\Phi(x, t)
$$

where $\Phi$ is an arbitrary function which depends on $w(x), q_{1, \ldots, n}(x)$ only. From $w$ norm conservation:

$$
\int_{-\infty}^{\infty} \Phi(x, t) d x=-\int_{-\infty}^{\infty} \frac{\partial w}{\partial t}(x, t) d x=-\frac{\partial}{\partial t} \int_{-\infty}^{\infty} w(x, t) d x=0
$$

If to substitute: $\Phi=\frac{\partial J}{\partial x}$ where $J(x)$ is some differentiable function, then eq. (3) demands:

$$
J(\infty, t)-J(-\infty, t)=0
$$

Let's suppose that such equality fulfilled, then $J(x)$ can be regarded as $w$ flow, and eq. (2) is equivalent to 1-dimensional flow continuity equation [13]:

$$
\frac{\partial w}{\partial t}=-\frac{\partial J}{\partial x}
$$


$J(x)$ can be decomposed formally as: $J(x)=w(x) v(x)$ where $v(x)$ corresponds to 1-dimensional $w$ flow velocity [13]; in such terms eq.(5) can be rewritten as:

$$
\frac{\partial w}{\partial t}=-v \frac{\partial w}{\partial x}-\frac{\partial v}{\partial x} w
$$

$v(x)$ presumably can be admitted as the independent $\mid g\}$ DF $q_{1}(x)$. Note that for normalized density $w(x, t)$ the condition of eq. (4) is trivial, in particular, it's fulfilled if $w$ flow $J(x, t)$ from/to $x= \pm \infty$ is negligible.

FM will be constructed here as minimal theory in a sense that at every step we shall choose its option with minimal number of DFs and theory constants. $m$ state representation in form of the line: $\mid g\}=\{w, v\}$ is asymmetric relative to its norm $w(x)$; besides, $w$ component evolution described by eq. (6) is nonlinear. Hence it's instructive to look for symmetric $\mid g\}$ representation $\eta(x)$ for which the state evolution would be linear, in principle, such $\eta$ ansatz can be complex, quaternionic, etc.. In general, it can be expressed as: $\eta(x)=\Upsilon_{x}(w, v)$ where $\Upsilon_{x}$ is some functional and $x$ is its parameter. However, $\eta$ norm corresponds to $w(x)$, hence if $w(x) \rightarrow 0$ for some $x \rightarrow x_{0}$, then $\eta(x)$ supposedly also should be negligible in $x_{0}$ vicinity. Hence $\eta$ can be decomposed also as:

$$
\eta(x)=\Upsilon_{x}(w, v)=f_{r}[w(x)] F_{x}(w, v)
$$

where $f_{r}$ is some real function, such that $f_{r}(\epsilon) \rightarrow 0$ for $\epsilon \rightarrow 0 ; F_{x}$ is an arbitrary functional. Because $\mid g\}$ is characterized by two DFs, it's worth to start $\eta$ ansatz search from complex $F_{x}$.

Plainly, $m$ evolution as the whole can be characterized by $m$ velocity $u(t)$ with expectation value $\bar{u}(t)$. Yet in FM, alike $m$ coordinate $x, u$ also can be also considered as fuzzy value $\tilde{u}(t)$ with the corresponding normalized distribution $w_{u}(u, t)$. Obviously, $\bar{u}(t)$ coincides with $\bar{v}(t)$ of (1), hence:

$$
\bar{u}=\int_{-\infty}^{\infty} v(x) w(x) d x
$$

i.e. its value is also defined by $w, v$. In place of $u$, below it will be convenient to use the variable $p=\mu u$ where $\mu$ is the theory constant; for the corresponding distribution $w_{p}(p)$, it gives $w_{u}(u)=\mu w_{p}(\mu u)$. We shall assume that analogously to QM, the expectation value of arbitrary $m$ observable $Q$ in FM is expressed as some $\mid g$ \} functional; in particular, $w_{p}(p)=F_{p}(\eta)$ where $F_{p}$ is the parameter dependent functional. The transformation $\eta \rightarrow w_{p}$ should possess the following properties:

i) Norm conservation: if $\eta(x)$ is normalized, then the same is true for $w_{p}$.

ii) $p$ expectation value $\bar{p}$ is expressed via $w(x), v(x)$ according to eq. (8).

iii) For free $m$ evolution $w_{p}$ is independent of $\eta(x) \rightarrow \eta(x+a)$ space shifts.

It can be proved that for complex $\eta(x)$ such properties have its fourier transform. To demonstrate it and calculate $w_{p}$, let's introduce the auxiliary form: $\varphi(p)=w_{p}^{\frac{1}{2}}(p) e^{i \beta(p)}$, here $\beta(p)$ is the dummy real function on which the final $w_{p}$ ansatz wouldn't depend. In accordance with eq. (7), $\eta(x)$ can be written as:

$$
\eta(x)=f_{r}(w) G_{x}(w, v) e^{i \lambda_{x}(w, v)}
$$

where $G_{x}(w, v), \lambda_{x}(w, v)$ are real functionals. Consider then $\varphi$ fourier decomposition on $X$ :

$$
\varphi(p)=\int_{-\infty}^{\infty} \eta(x) e^{-i p x} d x=\int_{-\infty}^{\infty} f_{r} G_{x} e^{i \lambda_{x}-i p x} d x
$$


For normalized $w_{p}$ the application of Plancherele formulae gives [12] :

$$
\int_{-\infty}^{\infty} w_{p}(p) d p=\int_{-\infty}^{\infty} \varphi(p) \varphi^{*}(p) d p=\int_{-\infty}^{\infty} f_{r}^{2}(w) G_{x}^{2} d x=1
$$

Application of du Bois-Raymond lemma [12] to the equality:

$$
\int_{-\infty}^{\infty}\left[f_{r}^{2}(w) G_{x}^{2}-w\right] d x=0
$$

permits to settle $G_{x}=1, f_{r}(w)= \pm w^{\frac{1}{2}}$. Then $\bar{p}$ can be calculated anew from 2-nd Plancherele formulae:

$$
\bar{p}=\int_{-\infty}^{\infty} p \varphi(p) \varphi^{*}(p) d p=\int_{-\infty}^{\infty} \frac{\partial \lambda_{x}}{\partial x} f_{r}^{2}(w) d x
$$

From the comparison with eq. (8), since $\bar{p}=\mu \bar{u}$ it follows: $\lambda_{x}=\gamma(x)+\chi(w)$ where $\gamma$ is $v$ functional:

$$
\gamma(x)=\mu \int_{-\infty}^{x} v(\xi) d \xi+c_{\gamma}
$$

here $c_{\gamma}$ is an arbitrary real number. $\chi(w)$ is an arbitrary real function which obeys to the condition:

$$
\int_{-\infty}^{\infty} w \frac{\partial \chi}{\partial x} d x=0
$$

If to choose $f=w^{\frac{1}{2}}$, then $w_{p}$ and $\beta(p)$ can be calculated from eq. (10) as functions of $\chi$, it's easy to check that $w_{p}$ is independent of $\beta(p)$, so $w_{p}$ is just $w, v$ functional. $\beta(p)$ is, in fact, the analogue of $\gamma(x)$ for $p$ observable. The resulting $m$ state in $x$-representation:

$$
\eta(x)=w^{\frac{1}{2}}(x) e^{i \gamma+i \chi}
$$

is the vector (ray) of complex Hilbert space $\mathcal{H}$. In this framework, the observable $p$ corresponds to the operator $\hat{p}=-i \frac{\partial}{\partial x}$ acting on $\eta$, i.e. $\bar{p}=\int \eta^{*} \hat{p} \eta d x$. It means that $x$ and $p$ observables are described by linear self-adjoint operators, which obey to the commutation relation $[\hat{x}, \hat{p}]=i$. By the analogy, we suppose that all $m$ observables $\{Q\}$ are the linear, self-adjoint operators on $\mathcal{H}$. If this is the case, $\eta(x)$ is the plausible candidate for $\{g\}$ state ansatz in $X$-representation, because for such $\eta$ the expectation values of all observables $\bar{Q}(t)$ expressed as semi-linear $\eta$ functionals.

Note that $\eta=e^{i \chi} g$, where $g(x, t)$ is standard QM wave function, so that $\eta(x, t)$ is its trivial map. Thus we shall study first $g(x, t)$ evolution, and then $\eta(x, t)$ properties will be derived from obtained results. Evolution equation for $g$ is supposed to be of the first order in time, i.e.:

$$
i \frac{\partial g}{\partial t}=\hat{H} g
$$

In general $\hat{H}$ is nonlinear operator, for the simplicity we shall consider first the linear case and turn to nonlinear one in the next section. The free $m$ evolution is invariant relative to $x$ space shifts performed 
by the operator $\hat{W}(a)=\exp \left(a \frac{\partial}{\partial x}\right)$ for arbitrary shift $a$. Because of it, corresponding free operator $\hat{H}_{0}$ should commute with $\hat{W}(a)$, i.e. $\left[\hat{H}_{0}, \frac{\partial}{\partial x}\right]=0$. It holds only if $\hat{H}_{0}$ is differential polinom of the form:

$$
\hat{H}_{0}=-\sum_{l=1}^{n} b_{l} \frac{\partial^{l}}{\partial x^{l}}
$$

where $b_{l}$ are arbitrary real constants, $n \geq 2$. From $X$-reflection invariance $b_{l}=0$ for noneven $l$. Suppose that the action of external field on $m$ can be accounted in $\hat{H}$ additively: $\hat{H}=\hat{H}_{0}+V(x, t)$ where $V$ is real nonsingular function. Let's rewrite eq. (17) separating $w, \gamma$ derivatives:

$$
i \frac{\partial g}{\partial t}=\left(i \frac{\partial w^{\frac{1}{2}}}{\partial t}-w^{\frac{1}{2}} \frac{\partial \gamma}{\partial t}\right) e^{i \gamma}=e^{i \gamma} \hat{Z} g
$$

where $\hat{Z}=e^{-i \gamma} \hat{H}$. Hence:

$$
\frac{\partial w^{\frac{1}{2}}}{\partial t}=i m(\hat{Z} g)
$$

Yet if to substitute $v(x)$ by $\gamma(x)$ in eq. (6) and transform it to $w^{\frac{1}{2}}$ time derivative, then:

$$
\frac{\partial w^{\frac{1}{2}}}{\partial t}=-\frac{1}{\mu} \frac{\partial w^{\frac{1}{2}}}{\partial x} \frac{\partial \gamma}{\partial x}-\frac{1}{2 \mu} w^{\frac{1}{2}} \frac{\partial^{2} \gamma}{\partial x^{2}}
$$

Plainly, the formalism consistency demands that the right side of this equation and $\operatorname{im}(\hat{Z} g)$ coincide for arbitrary $w, \gamma$, hence $\hat{H}$ can be obtained from their comparison term by term. In particular, the imaginary part of $\hat{Z} g$ includes the highest $\gamma$ derivative as the term $b_{n} w^{\frac{1}{2}} \frac{\partial^{n} \gamma}{\partial x^{n}}$, yet for eq. (21) the highest $\gamma$ derivative is proportional to $\frac{\partial^{2} \gamma}{\partial x^{2}}$. As the result, such comparison gives: $b_{2}=\frac{1}{2 \mu}$, for $l>2, b_{l}=0$, only in this case both expressions for $\frac{\partial w^{\frac{1}{2}}}{\partial t}$ would coincide. Thus $g$ free evolution is described by the only $\hat{H}_{0}$ term, so it follows that $\hat{H}$ is Schroedinger Hamiltonian for particle with mass $\mu$. The obtained ansatz gives also $J( \pm \infty, t)=0$ for $w$ flow of eq. (3), in accordance with our assumptions. Note that in standard QM $m$ evolution equation is postulated $a d$ hoc, here it's derived from FT premises for particle evolution on fuzzy manifold. The same is true for the commutation relation $[\hat{x}, \hat{p}]=i$

Plainly, $\gamma(x)$ corresponds to $\mid g\}$ quantum phase, so that:

$$
K_{1}\left(x, x^{\prime}\right)=\gamma(x)-\gamma\left(x^{\prime}\right)
$$

describes the phase correlation between the state components in $x, x^{\prime}$ analogous to $K_{0}\left(x, x^{\prime}\right)$ defined in sect. 1. It can be considered as the geometric structure which together with $w(x)$ defines the fuzzy state $\mid g\}$.

\section{General Fuzzy Dynamics}

In the previous section 1-dimensional FM formalism was derived from FT premises assuming that $\mid g\}$ evolution is linear and $\eta(x)$ gauge $\chi$ can be neglected. Here we shall drop both these assumptions one by one to obtain more general formalism. Concerning with nonlinear evolution, the conditions of QM linearity were reconsidered by Jordan, who shown that they are essentially weaker than Wigner theorem asserts [14]. In particular, if the evolution maps the set of all pure states one to one onto itself, and for arbitrary mixture of orthogonal states $\rho(t)=\sum P_{i}(t) \rho_{i}(t)$ all $P_{i}$ are independent of time, then such evolution is proved to be linear. Here $\rho_{i}(t)=g_{i}(t) g_{i}^{*}(t)$ are the density matrixes of orthogonal 
pure states $g_{i}$. Yet for considered FM formalism first condition is, in fact, generic: no mixed (i.e. probabilistic) state can appear in the evolution of pure fuzzy state. The second condition involves the probabilistic mixture of such orthogonal states and also seems to be rather weak assumption.

In addition, it follows that Jordan theorem permits to drop $\chi(w)$ defined in previous section. For the obtained $m$ state space defined by eq. (16) for all $\eta_{l}=g_{l} e^{i \chi}$ if $\left\langle g_{i} \mid g_{j}\right\rangle=\delta_{i j}$, then $\left\langle\eta_{i} \mid \eta_{j}\right\rangle=\delta_{i j}$ and vice versa. As was argued above, in FM any pure state $g\left(t_{0}\right)$ should evolve to pure state $g(t)$ for arbitrary $t$, so the same should be true for any $\eta\left(t_{0}\right)$. Now Jordan theorem can be applied to $\eta$ evolution, for that let's rewrite $g$ evolution equation for $\eta$ :

$$
i \frac{\partial g}{\partial t}=i \frac{\partial}{\partial t}\left(\eta e^{-i \chi}\right)=i \frac{\partial \eta}{\partial t} e^{-i \chi}+\eta \frac{\partial \chi}{\partial w} \frac{\partial w}{\partial t} e^{-i \chi}=\hat{H}\left(\eta e^{-i \chi}\right)
$$

From it one can come to the equation for $\eta$, the term containing $\frac{\partial w}{\partial t}$ can be rewritten according to (21). As the result, it gives:

$$
i \frac{\partial \eta}{\partial t}=e^{i \chi} \hat{H}\left(\eta e^{-i \chi}\right)+\frac{\eta}{\mu} e^{i \chi} \frac{\partial \chi}{\partial w} \frac{\partial}{\partial x}\left(w \frac{\partial \gamma}{\partial x}\right)
$$

Resulting equation for $\eta$ is also of first time order, but is openly nonlinear. Therefore, for arbitrary $\chi(w)$, given the initial $\eta\left(x, t_{0}\right)$, the resulting $\eta(x, t)$ can be only the equivalence class of $g(x, t)$ which evolves linearly from $g\left(x, t_{0}\right)=\eta\left(x, t_{0}\right) e^{-i \chi}$.

3-dimensional FM, in fact, doesn't demand any principal modification of described formalism. Analogously to 1-dimenisonal case, the fundamental set is $C^{F}=A^{F} \cup R^{3}$, hence for any fuzzy point $a_{j} \in A^{F}$ its properties should be defined relative to $X, Y, Z$ coordinate axes separately. Rotational symmetry implies that $a_{j}$ is described by the positive function $w^{j}(\vec{r})$ with norm $\int w^{j} d^{3} r=1$. If the particle $m$ corresponds to fuzzy point $a(t)$ characterized by $w(\vec{r}, t)$, then analogously to sect. 2 , given $w$ evolution depends on local parameters only, it can be described as:

$$
\frac{\partial w}{\partial t}(\vec{r}, t)=-\Phi(\vec{r}, t)
$$

where $\Phi$ is an arbitrary local function. Then from $w$ norm conservation 3-dimensional flow continuity equation follows:

$$
\frac{\partial w}{\partial t}=-\operatorname{div} \vec{J}
$$

One can decompose formally $\vec{J}=w \vec{v}$ and regard $w$ flow velocity $\vec{v}(\vec{r})$ as independent $\mid g$ \} parameter. $\mid g\}$ ansatz is supposed to be the complex $w, \vec{v}$ functional $\eta(\vec{r})$. Meanwhile, for $m$ as the whole, its velocity is supposedly characterized by the fuzzy vector $\vec{u}$ with the distribution $w_{u}(\vec{u})$ which obeys to the equation:

$$
\langle\vec{u}\rangle=\int \vec{u} w_{u}(\vec{u}) d^{3} u=\int \vec{v}(\vec{r}) w(\vec{r}) d^{3} r
$$

and $m$ kinematic fuzzy momentum defined as $\vec{p}=\mu \vec{u}$. From that, analogously to eqs. (8 - 16), the standard QM ansatz for $m$ state can be obtained: $g(\vec{r})=w^{\frac{1}{2}} e^{i \gamma}$ to which $\eta(\vec{r})$ is finally reduced. $g$ phase $\gamma(\vec{r})$ obeys to the equality $\mu \vec{v}=\operatorname{grad}(\gamma)$.

Considering $g$ linear evolution, for free $m$ evolution its operator $\hat{H}_{0}$ should be the even polinom of the form:

$$
\hat{H}_{0}=-\sum_{l=1}^{n} b_{2 l} \frac{\partial^{2 l}}{\partial \vec{r}^{2 l}}
$$

If the external field action can be described by the addition of real function $V$ to it:

$$
\hat{H}=\hat{H}_{0}+V(\vec{r}, t)
$$


then from $\frac{\partial g}{\partial t}$ the term $\frac{\partial w^{\frac{1}{2}}}{\partial t}$ can be extracted and expressed via $w, \gamma \vec{r}$-derivatives. From their comparison with corresponding $\hat{H} g$ derivatives the Schroedinger equation is obtained for $m$ evolution. The applicability of Jordan theorem to 3-dimensional $\hat{H}$ is obvious, because the derivation of $\hat{H}$ linearity doesn't depend on the dimensiality of coordinate space. The same is true for the proof of uniqueness of $g(\vec{r}, t)$ ansatz, i.e. that $\chi(w)=0$. We shall admit that in FM observer can prepare an arbitrary initial state $g_{0}(x)$, such assumption corresponds to the superposition principle if $g_{0}=g_{1}+g_{2}$ and $g_{1,2}$ are arbitrary states with the proper norms. Planck constant $\hbar=1$ in our FM ansatz, but the same value ascribed to it in relativistic unit system together with velocity of light $c=1$; in FM framework $\hbar$ only connects $x, p$ geometric scales and doesn't have any other meaning.

In our derivation of evolution equation we didn't assume Galilean invariance of FM, rather in our approach it follows from the obtained equation, if the reference frames (RFs) are regarded as the physical objects with mass $\mu \rightarrow \infty$ [6]. For the transition to relativistic FM from our ansatz its natural extension for complex scalar state $g$ is Klein-Gordon equation. Yet for such equation it's impossible to define $m$ density $\rho(\vec{r})$ ansatz such that $\rho(\vec{r})$ will be nonnegative for all free states [11]. As was argued in sect. 2, in general, $g$ scalar ansatz can be complex, quaternionic and so on. Hence to cope with described difficulty, in Klein-Gordon equation the complex $g$ can be substituted by the quaternion scalar $\xi(\vec{r}, t)$, which gives:

$$
\left(\frac{\partial^{2}}{\partial t^{2}}-\Delta+\mu^{2}\right) \xi=0
$$

For such state the single quantum phase $i \gamma$ is extended to three independent phases $i \gamma+j \beta+k \alpha$ which correspond to additional geometric DFs. Such DFs can be considered as $m$ phase correlations $K_{1,2,3}$ analogous to (22). To get nonnegative $\rho$ one should broke first $i, j, k$ space symmetry and to choose an arbitrary orthogonal basis $i^{\prime}, j^{\prime}, k^{\prime}$. Plainly, in this basis $\xi=\psi_{1}+\psi_{2} j^{\prime}$ where $\psi_{1,2}=a_{1,2}+b_{1,2} i^{\prime}$ and $a_{1,2}, b_{1,2}$ are real functions. Let's write $\psi_{1,2}$ as the spinor $\psi_{u}$ and define the auxilary spinor:

$$
\psi_{d}=-i^{\prime}\left(I \frac{\partial}{\partial t}+\frac{\partial}{\partial \vec{r}} \vec{\sigma}\right) \psi_{u}
$$

in obvious notations. If to denote $\psi_{d}$ components as $\psi_{3,4}$, then it's easy to check that $\rho(\vec{r})=\sum_{1}^{4}\left|\psi_{l}\right|^{2}$ will be nonnegative and normalizable for arbitrary $\xi$. Furthermore, if to regard $\psi_{1, \ldots, 4}$ as 4 -spinor components, then such 4-spinor would obey to Dirac equation in chiral representation [15]. Thus, in FM some geometric DFs defined on fuzzy manifold can be 'compactified', resulting in the appearance of internal spinor space, so that the particle $m$ acquires spin $\frac{1}{2}$.

Now we shall consider the interaction between fuzzy states in FM framework. As follows from eq.( $18-21) m$ free dynamics can be described by the system of two equations which define $\frac{\partial w^{\frac{1}{2}}}{\partial t}$ and $\frac{\partial \gamma}{\partial t}$ which for 3-dimensions are equal to:

$$
\begin{gathered}
\frac{\partial w^{\frac{1}{2}}}{\partial t}=-\frac{1}{\mu} \frac{\partial w^{\frac{1}{2}}}{\partial \vec{r}} \frac{\partial \gamma}{\partial \vec{r}}-\frac{1}{2 \mu} w^{\frac{1}{2}} \frac{\partial^{2} \gamma}{\partial \vec{r}^{2}} \\
\frac{\partial \gamma}{\partial t}=-\frac{1}{2 \mu}\left[\left(\frac{\partial \gamma}{\partial \vec{r}}\right)^{2}-\frac{1}{w^{\frac{1}{2}}} \frac{\partial^{2} w^{\frac{1}{2}}}{\partial \vec{r}^{2}}\right]
\end{gathered}
$$

Yet the first of them is equivalent to eq. (26) which describes just $w(\vec{r})$ balance and so is, in fact, kinematical one and can't depend on any interactions directly. Namely, under some external influence the values of $w, \gamma$ variables can change, but no new terms can appear in that equation. Note that in nonrelativistic QED $e \vec{A}$ term formally appears in it, but it's just the part of the expression for kinematic momentum [11]. Hence $m$ interactions can be accounted only via the modification of second equation 
of this system. Assuming that analogously to eq. (29) the evolution terms are additive, it gives: $\hat{H}=\hat{H}_{0}+\hat{H}_{\text {int }}$ where $\hat{H}_{\text {int }}$ is the interaction term. Consider how the interaction of two particles $m, M$ can be described in such approach. Let suppose that $m, M$ interaction is universal in a sense that $\left\langle\hat{H}_{\text {int }}\right\rangle \neq 0$ for arbitrary relative $m, M$ momentum $\left\langle\vec{p}_{12}\right\rangle$, and is induced by the conserved charges $q_{1}, q_{2}$. Then the main $\hat{H}_{\text {int }}$ term which survives at $\left\langle\vec{p}_{12}\right\rangle \rightarrow 0$ is equal to $q_{1} q_{2} U\left(r_{12}\right)$. Obtained $U\left(r_{12}\right)$ corresponds to the classical potential, yet in standard QM such interaction is, in fact, postulated from classical-to-quantum correspondence, whereas here it follows from FM geometric premises. Since $\gamma$ corresponds to the quantum phase, it supposes that in FM $m$ interactions can possess some form of local gauge invariance [16]. Of course, one can just postulate the gauge interactions of certain kind, yet is seems worth to explore whether such dynamics can be obtained from some considerations related to FM or some other fundamental principles.

In conclusion, we have shown that the quantization of elementary systems can be derived directly from axiomatic of Set theory and topology together with the natural assumptions about system evolution. It allows to suppose that the quantization phenomenon has its roots in foundations of mathematics [11]. Our approach permits to construct QM formalism starting from geometric concepts and structures only, so in these aspects it's analogous to general relativity construction. In the same time the considered fuzzy manifold describes the possible variant of fundamental pregeometry which is basic component of some quantum gravity theories [5]. In this vein, FM provides the interesting opportunities, being generically nonlocal theory which, in the same time, can possess Lorentz covariance and local gauge invariance.

\section{References}

[1] Marmo, G., Volkert G., Phys. Scr. 82, 038117-038128 (2010)

[2] Dodson C., Bull. London Math. Soc., 6, 191-196 (1974)

[3] Zeeman C. in: Topology of 3-manifolds, , ed. K. Fort (Prentice-Hall, New Jersey, 1961) 240-248

[4] Sostak A., J. Math. Sci. 6, 662-691 (1996)

[5] Balachandran A., Kurkcuoglu S., Vaidia S. Lectures on Fuzzy and Fuzzy SUSY Physics (World Scientific, Singapour, 2007) 32-121

[6] Mayburov S., J. Phys. A41., 164071-164080 (2007)

[7] Mayburov S., Int. J. Theor. Phys. 49, 3192-3198 (2009)

[8] Mayburov S., Int. J. Theor. Phys (2015) to be published; Quant-ph: 1205.3019

[9] Schroder B. Ordered Sets: An Introduction (Boston, Birkhauser,2003)

[10] Bandemer H., Gottwald S. Fuzzy sets, Fuzzy Logics, Fuzzy Methods with Apllications (Wiley, N-Y, 1995)

[11] Jauch J. Foundations of Quantum Mechanics. (Reading, Addison-Wesly, 1968)

[12] Reed M., Simon B. Methods of Modern Mathematical Physics (N-Y, Academic Press, 1972)

[13] Landau L., Lifshitz E. Mechanics of Continuous Media (Oxford; N.Y., Pergamon Press, 1976) 6-32

[14] Jordan T., Phys. Rev. A73, $022101-022109$ (2006)

[15] Fayyazuddin A., Riazuddin B.Quantum Mechanics (W. S., Singapore, 1990) 445-461

[16] Cheng T., Li L. Gauge Theory of Elementary Particles. (Claredon, Oxford, 1984) 151-194 